\title{
AVALIAÇÃO DOS FATORES DE CANCELAMENTO DE CIRURGIAS EM HOSPITAIS DO NORDESTE BRASILEIRO
}

\author{
Evaluation of cancellation factors of surgeries in northeast brazilian hospitals
}

\section{Evaluación de factores de cancelación de cirugía en hospitales brasileños del noreste}

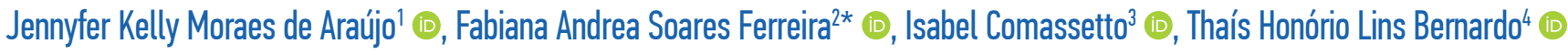

RESUMO: Objetivos: Identificar a taxa de cancelamento cirúrgico e avaliar os fatores que causam o cancelamento de cirurgias. Método: Pesquisa quantitativa realizada nos centros cirúrgicos de dois hospitais de grande porte do Nordeste brasileiro, sendo um público e o outro privado-público. A pesquisa foi aprovada pelo Comitê de Ética, sob Certificado de Apresentação para Apreciação Ética (CAAE) 46831515.6.0000.5013, e a coleta dos dados foi realizada por meio do banco de dados eletrônico dos hospitais. Resultados: No período de janeiro de 2014 a dezembro de 2015, no hospital privado-público, foram programadas 20.810 cirurgias e suspensas 4.815, com taxa de suspensão cirúrgica de 23,3\%. No hospital público, foram programadas 6.392 cirurgias e suspensas 2.702 , com taxa de suspensão de 42,7\%. Os principais fatores de suspensão relacionaram-se ao paciente e a aspectos administrativos. Conclusão: Foi possível identificar aumento da incidência de cancelamentos cirúrgicos no decorrer dos anos, evidenciando taxas de suspensão consideráveis. Dessa forma, é preciso que as instituições busquem estratégias com a finalidade de minimizar a incidência de cancelamentos de procedimentos anestésico-cirúrgicos nas instituições de saúde. Palavras-chave: Avaliação em saúde. Centro cirúrgico hospitalar. Enfermagem perioperatória. Hospitais.

ABSTRACT: Objectives: To identify the surgical cancellation rate and to evaluate the factors that cause surgery cancellation. Methodology: Quantitative research conducted in the surgical centers of two large hospitals, one public and one private-public, in the Northeast of Brazil. The research was approved by the Ethics Committee, under Certificate of Presentation for Ethical Appraisal (CAAE) 46831515.6.0000.5013, and data collection was performed through the electronic database of the hospitals. Results: From January 2014 to December 2015, in the public-private hospital, 20,810 surgeries were scheduled and 4,815 were suspended, with a surgical suspension rate of $23.3 \%$. In the public hospital, 6,392 surgeries were scheduled and 2,702 suspended, with a suspension rate of $42.7 \%$. The main suspension factors were related to patients and administrative aspects. Conclusion: It was possible to identify increased incidence of surgical cancellations over the years, showing considerable suspension rates. Thus, institutions need to seek strategies to minimize the incidence of cancellations of anesthetic-surgical procedures in health institutions. Keywords: Health evaluation. Surgery department, hospital. Perioperative nursing. Hospitals.

RESUMEN: Objetivos: Identificar la tasa de cancelación quirúrgica y evaluar los factores que causan la cancelación de la cirugía. Método: investigación cuantitativa realizada en los centros quirúrgicos de dos grandes hospitales en el noreste de Brasil, uno público y otro privado-público. La investigación fue aprobada por el Comité de Ética, bajo el Certificado de Presentación para la Evaluación Ética (CAAE) 46831515.6.0000.5013, y la recopilación de datos se realizó a través de la base de datos electrónica de hospitales. Resultados: desde enero de 2014 hasta diciembre de 2015, en el hospital público-privado, se programaron 20.810 cirugías y se suspendieron 4.815, con una tasa de suspensión quirúrgica del 23,3\%. En el hospital público, se programaron 6.392 cirugías y se suspendieron 2.702 , con una tasa de suspensión del 42,7\%. Los principales factores de suspensión estaban relacionados con el paciente y los aspectos administrativos. Conclusión: fue posible identificar una mayor incidencia de cancelaciones quirúrgicas a lo largo de los años, mostrando tasas de suspensión considerables. Por lo tanto, las instituciones deben buscar estrategias para minimizar la incidencia de cancelaciones de procedimientos anestésico-quirúrgicos en instituciones de salud. Palabras clave: Evaluación en salud. Servicio de cirugía en hospital. Enfermería perioperatoria. Hospitales.

'Enfermeira pela Escola de Enfermagem da Universidade Federal de Alagoas (UFAL) - Maceió (AL), Brasil.

2Enfermeira; mestre em Saúde do Adulto pela Universidade de São Paulo (USP). Docente em Enfermagem da UFAL - Maceió (AL), Brasil.

${ }^{3}$ Enfermeira; doutora em ciências pela USP - São Paulo (SP), Brasil.

«Enfermeira; doutora em Biotecnologia pela Rede Nordeste de Biotecnologia (RENORBIO). Docente em Enfermagem da UFAL - Maceió (AL), Brasil.

${ }^{*}$ Autora correspondente: thais.bernardo@esenfar.ufal.br

Recebido: 31/07/2018 - Aprovado: 01/09/2019

DOI: $10.5327 / Z 1414-4425201900040002$ 


\section{INTRODUÇÃO}

O aumento da taxa de cancelamento de cirurgias prejudica o planejamento e a organização do serviço de saúde, além de acarretar repercussões aos pacientes, aos profissionais e às instituição de saúde ${ }^{1}$. Essa ocorrência nem sempre recebe a devida atenção por parte da equipe de saúde nem da administração do próprio hospital².

A suspensão da cirurgia oferece transtornos para o paciente, podendo causar abalos psicológicos e prejuízo no planejamento da vida diária, bem como para o hospital, gerando atrasos na programação cirúrgica, prejuízos para outros pacientes, ampliação do custo operacional e financeiro, prolongamento do período de internação e aumento do risco de infecções ${ }^{3}$. Quanto ao impacto emocional e aos custos, ambos podem ser minimizados se a suspensão for feita antes da internação ${ }^{4}$.

A melhoria da qualidade dos serviços de saúde vem sendo promovida por meio do emprego dos indicadores de qualidade utilizados para monitorar e avaliar a eficiência assistencial e as atividades de um serviço de acordo com suas características 5 . A quantidade de cirurgias canceladas é uma variável que interfere no desempenho dos indicadores quantitativos, constituindo parâmetros de avaliação de produtividade e qualidade dos serviços, sendo a taxa de suspensão de cirurgia um deles ${ }^{6}$.

"A taxa de suspensão cirúrgica é definida pelo número de procedimentos cancelados, dividido pelo total de cirurgias programadas em um período de tempo, multiplicado por cem"3. Portanto, o padrão do serviço e a melhoria dos indicadores de qualidade, como a redução da taxa de cancelamento de cirurgias, necessitam de melhor gerenciamento de fluxos, de mais informações e de agendamentos dos procedimentos, para melhor identificação da taxa de suspensão ${ }^{6}$.

Enfermeiros de centro cirúrgico (CC) têm seu trabalho voltado para administrar e gerenciar a unidade e provê-la de equipamentos, com a finalidade de propiciar as melhores circunstâncias para o desenvolvimento do processo cirúrgico, para melhoria da condição de saúde dos pacientes. Para tanto, realizam o planejamento e precisam da programação das equipes cirúrgicas e dos outros profissionais ou serviços envolvidos e, diante das intercorrências, contam com certa previsibilidade em CC de grande porte ${ }^{2}$.

O enfermeiro coordenador é responsável pelo controle administrativo, técnico-operacional e ético, pela provisão e previsão de recursos humanos e materiais adequados, pelo planejamento estratégico de enfermagem, pela verificação do agendamento no mapa cirúrgico e pela orientação da montagem das salas operatórias (SO), entre outras atividades. Enfermeiros assistenciais são responsáveis pela checagem da programação cirúrgica, supervisão da atuação da equipe de enfermagem, elaboração da escala de atividades diárias dos profissionais, entre outras tarefas ${ }^{7}$.

Diante do exposto, percebe-se que o cancelamento de cirurgias e sua taxa de suspensão são temáticas em evidência nos estudos, e essa predominância no serviço de saúde interfere na qualidade da assistência. Portanto, é preciso que as instituições busquem medidas que favoreçam o melhor gerenciamento da rotina do CC, o levantamento de dados, o planejamento das ações e a contratação de enfermeiros capacitados para promover assistência adequada e humanizada, capazes de desenvolver estratégias que possam minimizar os cancelamentos, com os intuitos de melhorar a qualidade da assistência em saúde e reduzir a taxa de suspensão de procedimentos anestésico-cirúrgicos.

\section{OBJETIVOS}

- Identificar a taxa de cancelamento cirúrgico em dois hospitais situados na Região Nordeste do Brasil;

- Avaliar os fatores que causam cancelamento de cirurgias nesses dois hospitais.

\section{MÉTODO}

Trata-se de uma pesquisa quantitativa e descritiva aprovada pelo Comitê de Ética em Pesquisa da Universidade Federal de Alagoas, Alagoas, Brasil, sob Certificado de Apresentação para Apreciação Ética (CAAE) número 46831515.6.0000.5013, realizada de janeiro de 2014 a dezembro de 2015, nos CC de dois hospitais de grande porte localizados em uma região do Nordeste brasileiro, sendo um hospital privado-público e o outro público.

Antes de começar a coleta de dados, o pesquisador apresentou-se aos diretores dos hospitais, juntamente com o projeto de pesquisa, com a finalidade de solicitar a autorização para o estudo. Ao ser formalizada a autorização da instituição, deu-se início à coleta dos dados.

A coleta dos dados foi realizada por meio do banco de dados eletrônico, no qual foi possível obter as informações pertinentes ao quantitativo de cirurgias programadas e suspensas, gerando taxa de suspensão cirúrgica mensal e anual, de modo 
a identificar os fatores que causaram as suspensões cirúrgicas e as especialidades médicas que mais suspendem cirurgias, além de analisar o sexo e o convênio dos pacientes.

Os dados coletados foram tabulados no programa Excel, do Microsoft Office ${ }^{\circledR}$, e a análise ocorreu mediante frequências absolutas, representadas na forma de tabelas e gráficos.

\section{RESULTADOS}

No hospital privado-público, entre 2014 e 2015, foram programadas 20.810 cirurgias e canceladas 4.815 , apontando taxa de suspensão cirúrgica de $23,3 \%$. Durante esse período, a taxa de suspensão apresentou variação de 16,9 a $41,8 \%$, considerando-se as estatísticas mensais. Como evidenciado na Tabela 1, por alguns meses a taxa de suspensão se manteve na mesma proporção nos dois anos. No entanto, em 2014, as menores taxas foram nos meses de agosto $(17,4 \%)$ e janeiro e setembro (20,4\%), e, em 2015, em janeiro (16,9\%) e novembro $(18,4 \%)$. Já as maiores taxas, em 2014, foram nos meses de dezembro $(25,4 \%)$ e março $(26,4 \%)$, e em 2015 , nos meses de outubro $(26,8 \%)$ e dezembro $(41,8 \%)$.

Em comparação com o mesmo período de 2014, em 2015, a partir do mês de agosto, houve elevação das taxas de suspensão cirúrgica. Nesse período, no hospital privado-público, viu-se redução da quantidade de cirurgias programadas, mas o quantitativo de suspensões permaneceu, e o mês de dezembro apresentou maior número de cirurgias suspensas, revelando taxa de suspensão de $41,8 \%$.

No hospital público, nos dois anos, programaram-se 6.392 cirurgias e 2.702 foram suspensas, indicando taxa de suspensão de $42,27 \%$. Conforme a Tabela 2 , no decorrer do período estudado, no hospital público houve variação da taxa de suspensão cirúrgica de 3,79 a 115,53\%. As menores taxas no ano de 2014 foram nos meses de setembro (3,79\%), maio (28,94\%) e agosto (29,01\%), e, em 2015 , em dezembro $(8,05 \%)$ e novembro $(22,71 \%)$. Já as maiores taxas de suspensão em 2014 ocorreram em novembro (72,88\%) e outubro $(42,58 \%)$, e no ano de 2015 , em setembro $(115,53 \%)$ e fevereiro $(75,84 \%)$.

Em 2015, foram reveladas as maiores taxas de suspensões cirúrgicas, com destaque para o mês de setembro, que obteve incidência de $115,53 \%$. Na análise dos dados, verificou-se que a quantidade de cirurgias suspensas foi maior que a programada. Por isso, a taxa de suspensão foi elevada, maior do que $100 \%$.

Esse fato decorreu uma vez que no hospital público ocorrem encaixes de procedimentos na programação cirúrgica, que chegam ao CC no mesmo dia, após a impressão do mapa cirúrgico. Esses encaixes são referentes a cirurgias em que o paciente chega ao hospital no dia da operação, sem ter realizado a confirmação cirúrgica.

Tabela 1. Cirurgias programadas e suspensas e taxa de suspensão cirúrgica do hospital privado-público, de acordo com os meses dos anos, em 2014 e em 2015*.

\begin{tabular}{|l|c|c|c|c|c|c|}
\hline Mês & Programadas & Suspensas & $\begin{array}{c}\text { Taxa de } \\
\text { suspensão } \\
\text { cirúrgica (\%) }\end{array}$ & Programadas & Suspensas & $\begin{array}{c}\text { Taxa de } \\
\text { suspensão } \\
\text { cirúrgica (\%) }\end{array}$ \\
\hline Janeiro & 815 & 166 & 20,4 & 847 & 143 & 16,9 \\
\hline Fevereiro & 852 & 179 & 21,0 & 690 & 170 & 24,6 \\
\hline Março & 810 & 214 & 26,4 & 1.059 & 235 & 22,2 \\
\hline Abril & 880 & 198 & 22,5 & 899 & 209 & 23,5 \\
\hline Maio & 753 & 192 & 25,5 & 937 & 238 & 25,4 \\
\hline Junho & 866 & 215 & 24,8 & 949 & 217 & 22,9 \\
\hline Julho & 1013 & 226 & 22,4 & 1.028 & 220 & 21,4 \\
\hline Agosto & 903 & 157 & 17,4 & 787 & 204 & 25,9 \\
\hline Setembro & 916 & 187 & 20,4 & 863 & 213 & 24,7 \\
\hline Outubro & 1.057 & 226 & 21,4 & 559 & 150 & 26,8 \\
\hline Novembro & 942 & 196 & 20,8 & 806 & 148 & 18,4 \\
\hline Dezembro & 905 & 230 & 25,4 & 674 & 282 & 41,8 \\
\hline
\end{tabular}


Sendo assim, a cirurgia passa a ser contabilizada como realizada ou suspensa para o controle de gestão hospitalar e não como programada, e, então, o quantitativo de cirurgias suspensas pode ser maior do que o das programadas, evidenciando altas taxas de suspensão. No período estudado, obteve-se diferença no quadro da programação cirúrgica, pois o quantitativo de cirurgias programadas no hospital privado-público foi três vezes maior do que no hospital público, porém o hospital público, mesmo apresentando quantidade menor de programação cirúrgica, obteve maior número de suspensão cirúrgica e exibiu a maior taxa, igual a $42,27 \%$.

Em relação ao sexo biológico, no hospital privado-público, do total das 4.815 cirurgias suspensas, 2.371 (49,3\%) pacientes eram do sexo feminino e 2.444 (50,8\%) do masculino. No hospital público, do total das 2.702 cirurgias suspensas, $1.592(58,91 \%)$ pacientes eram do sexo feminino e 971 (35,93\%) do masculino, sendo, portanto, a maioria do sexo feminino.

Quanto ao convênio, no hospital privado-público, em 2014 , do total de 2.386 (22,3\%) cirurgias suspensas, 882 $(36,9 \%)$ foram particulares e/ ou de plano de saúde e 1.504 $(63,1 \%)$ pelo Sistema Único de Saúde (SUS). No ano de 2015, das 2.429 cirurgias suspensas, $874(35,9 \%)$ eram particulares e/ ou de plano de saúde e 1.555 (64.01\%) eram do SUS. Nesse contexto, percebe-se que a maior quantidade das suspensões foi das cirurgias conveniadas pelo SUS.
Os fatores que causaram suspensão dos procedimentos cirúrgicos foram agrupados e identificados neste estudo por:

- Assistenciais: falta de jejum e ausência de exames complementares (hospital privado-público e hospital público);

- Administrativos: falta de material, carência de médicos, ausência de sangue e hemoderivados, inexistência de medicação, substituição por outro paciente de urgência, suspensão pelo cirurgião, erro na marcação (em ambos os hospitais), cirurgia remarcada (apenas no hospital privado-público), falta de membro da equipe de enfermagem e não liberação da autorização de internação hospitalar (apenas no hospital público);

- Estruturais da instituição: ausência de leito e de vaga na unidade de terapia intensiva (UTI) (em ambos os hospitais), indisponibilidade de $\mathrm{SO}$ e equipamento em manutenção (apenas no hospital público);

- Relacionados ao paciente: não comparecimento, falta de condições clínicas, cirurgia já realizada e óbito (ambos os hospitais).

- Outros: falta de tempo hábil (ocorre quando as cirurgias têm o tempo de realização prolongado, ocasionando o atraso de algumas e suspensão de outras, por falta de tempo no dia para a realização de outros procedimentos cirúrgicos) e não internação do paciente, em razão da não autorização do convênio (hospital público-privado e público).

Tabela 2. Cirurgias programadas e suspensas e taxa de suspensão cirúrgica do hospital público, de acordo com os meses dos anos, em 2014 e em 2015*.

\begin{tabular}{|c|c|c|c|c|c|c|}
\hline \multirow[b]{2}{*}{ Mês } & \multicolumn{3}{|c|}{2014} & \multicolumn{3}{|c|}{2015} \\
\hline & Programadas & Suspensas & $\begin{array}{c}\text { Taxa de } \\
\text { suspensão } \\
\text { cirúrgica (\%) }\end{array}$ & Programadas & Suspensas & $\begin{array}{c}\text { Taxa de } \\
\text { suspensão } \\
\text { cirúrgica (\%) }\end{array}$ \\
\hline Janeiro & 168 & 53 & 31,54 & 242 & 134 & 55,37 \\
\hline Fevereiro & 219 & 81 & 36,98 & 178 & 135 & 75,84 \\
\hline Março & 201 & 64 & 31,84 & 322 & 135 & 41,92 \\
\hline Abril & 252 & 88 & 34,92 & 301 & 73 & 24,25 \\
\hline Maio & 304 & 88 & 28,94 & 275 & 119 & 43,27 \\
\hline Junho & 242 & 86 & 35,53 & 268 & 97 & 36,19 \\
\hline Julho & 271 & 104 & 38,37 & 322 & 105 & 32,60 \\
\hline Agosto & 293 & 85 & 29,01 & 314 & 150 & 47,77 \\
\hline Setembro & 316 & 12 & 3,79 & 206 & 238 & 115,53 \\
\hline Outubro & 364 & 155 & 42,58 & 241 & 170 & 70,53 \\
\hline Novembro & 284 & 207 & 72,88 & 317 & 72 & 22,71 \\
\hline Dezembro & 258 & 90 & 34,88 & 273 & 22 & 8,05 \\
\hline
\end{tabular}

*\%: taxa de suspensão cirúrgica. 
Os principais fatores que causaram cancelamento no hospital privado-público e no hospital público, nos dois anos analisados, foram concernentes ao paciente. Entre esses fatores, destacam-se: não comparecimento do paciente, com 1.145 ocorrências (47,9\%) em 2014 e 1.207 (42,5\%) em 2015 no hospital privado-público; e 463 (41,33\%) em 2014 e 425 (26,9\%) em 2015 no hospital público. Já as suspensões por falta de condições clínicas do paciente foram: 275 (11,5\%) em 2014 e 274 (11,3\%) em 2015 no hospital privado-público; e 220 (19,6\%) em 2014 e 390 (24,7\%) em 2015 no hospital público.

Em relação aos fatores administrativos, a falta de material e de médicos foi destaque em ambos os hospitais. No hospital privado-público e no hospital público, respectivamente, a falta de material correspondeu a $128(5,4 \%)$ e $56(5,0 \%)$ cirurgias suspensas em 2014; $169(6,9 \%)$ e $150(14,3 \%)$ em 2015; e por carência de médicos, 127 (5,3\%) e 134 (11,7\%) em 2014 e 175 $(7,2 \%)$ e 137 (8,7\%) em 2015. Outro fator em evidência no hospital público em 2015 foi a falta de medicação, que representou $121(7,64 \%)$ suspensões. Entretanto, em ambos os anos, foram constatados cancelamentos por outros fatores, como: $293(12,1 \%)$ e $310(12,8 \%)$ por falta de tempo hábil e 85 (3,5\%) por paralisação do SUS no ano de 2015. A Tabela 3 traz as principais causas de cancelamento de cirurgias nos dois hospitais.

Conforme destacado na Figura 1, os fatores pertinentes ao paciente foram os mais frequentes em ambos os hospitais. Em seguida, estão os administrativos, com maior proporção no hospital público, porém o hospital privado-público destacou-se em outros fatores de suspensão, como falta de tempo hábil e paralisação do SUS.

As especialidades médicas que tiveram maior número de suspensões no hospital privado-público em 2014 e 2015 foram, respectivamente: ortopedia, com $825(34,6 \%)$ e 645 (22,7\%); cirurgia geral, com 413 (17,3\%) e 468 (19,6\%); otorrinolaringologia, com 208 (8,7\%) e 257 (10,7\%); pediatria, com 178
$(7,7 \%)$ e $159(6,3 \%)$; urologia, com $137(5,7 \%)$ e $128(5,3 \%)$; e ginecologia, com 125 (5,2\%) e 185 (7,7\%).

No hospital público, as especialidades com maior incidência de cancelamento em 2014 e 2015 foram, respectivamente: oftalmologia, com $404(36,07 \%)$ e $561(35,46 \%)$; cirurgia geral, com $158(14,10 \%)$ e 201 (12,70\%); cirurgia plástica, com $113(10,08 \%)$ e 93 (5,87\%); urologia, com $65(5,80 \%)$ e 137 (8,65\%); ginecologia, com 90 (8,03\%) e 101 (6,38\%); e pediatria, com 87 (7,76\%) e 91 (5,75\%).

A Figura 2 mostra a comparação da taxa de suspensão de cirurgias nos dois hospitais, de acordo com a especialidade. Observa-se que os maiores índices de suspensão do hospital privado-público foram nas especialidades de ortopedia e cirurgia geral e do hospital público foram oftalmologia e cirurgia geral.

Em virtude dos resultados encontrados, foi realizada avaliação dos fatores que causaram maiores índices de suspensão, segundo especialidades, nos dois hospitais. No hospital privado-público, o principal fator de suspensão das especialidades de ortopedia e cirurgia geral foi o não comparecimento do paciente, seguido da falta de tempo hábil, pois, do total das suspensões de cada especialidade, 1.498 e 879, em 2014 e 2015, foram 428 (28,57\%) e 349 (39,70\%) suspensões por não comparecimento do paciente; e $304(20,29 \%)$ e $122(13,87 \%)$ por falta de tempo hábil.

Além dessas causas, é importante salientar que os achados mostram a ocorrência de suspensões na área de ortopedia em razão da falta de material, na proporção de 165 $(11,01 \%)$ cirurgias canceladas. Já na especialidade de otorrinolaringologia, com total de 436 suspensões, os principais fatores foram o não comparecimento do paciente e a falta de condições clínicas, que representaram 314 (72,01\%) e 60 $(13,76 \%)$ suspensões, respectivamente.

No hospital público, das especialidades oftalmologia (959), cirurgia geral (359) e cirurgia plástica (206), os fatores de suspensão mais recorrentes da das duas primeiras foram:

Tabela 3. Principais fatores que causaram suspensão cirúrgica no hospital privado-público e no hospital público, nos anos 2014 e 2015 *

\begin{tabular}{|c|c|c|c|c|c|c|c|c|c|}
\hline \multirow{3}{*}{ Fatores } & & \multicolumn{4}{|c|}{ Hospital privado-público } & \multicolumn{4}{|c|}{ Hospital público } \\
\hline & & \multicolumn{2}{|c|}{2014} & \multicolumn{2}{|c|}{2015} & \multicolumn{2}{|c|}{2014} & \multicolumn{2}{|c|}{2015} \\
\hline & & N. & $\%$ & N. & $\%$ & $\mathbf{N .}$ & $\%$ & $\mathbf{N .}$ & $\%$ \\
\hline \multirow{2}{*}{$\begin{array}{l}\text { Relacionados ao } \\
\text { paciente }\end{array}$} & $\begin{array}{c}\text { Não comparecimento } \\
\text { do paciente }\end{array}$ & 1.145 & 47,9 & 1.270 & 42,5 & 463 & 41,33 & 425 & 26,9 \\
\hline & $\begin{array}{c}\text { Falta de } \\
\text { condições clínicas }\end{array}$ & 275 & 11,5 & 274 & 11,3 & 220 & 19,6 & 390 & 24,7 \\
\hline \multirow{2}{*}{ Administrativos } & Falta de material & 128 & 5,4 & 169 & 6,9 & 56 & 5,0 & 150 & 14,3 \\
\hline & Carência de médicos & 127 & 5,3 & 175 & 7,2 & 134 & 11,7 & 137 & 8,7 \\
\hline
\end{tabular}

*\%: taxa de suspensão cirúrgica. 
não comparecimento do paciente, com 537 (55,99\%) e 75 (20,89\%); e falta de condições clínicas do paciente, com 233 $(24,29 \%)$ e 68 (18,9\%). Quanto à cirurgia plástica, houve divergência no comparativo da frequência dos fatores, pois a falta de condições clínicas apresentou o maior quantitativo, $51(24,75 \%)$, em relação a $49(23,78 \%)$ por falta do paciente.

\section{DISCUSSÃO}

Atualmente, o cancelamento de cirurgias é considerado um dos indicadores de qualidade da assistência, sendo analisado por meio da taxa de suspensão cirúrgica ${ }^{8}$. Nesta pesquisa, a taxa de suspensão, no período estudado, no hospital privado-público foi de $23,13 \%$, e no hospital público, de 42,27\%. Em comparação com a pesquisa, estudos revelam a existência de variação das taxas entre $33,8^{9}, 18,45^{5}, 17,3^{10}$ e $13,60 \%{ }^{3}$.

Em relação à análise das taxas de suspensão mensal da pesquisa, houve divergência com estudo realizado no CC de um hospital universitário do Paraná. O estudo ocorreu no período de oito meses, com 2.828 cirurgias programadas, 522 suspensas e taxa de suspensão de 18,45\%, evidenciando frequência da taxa variável durante os meses. Em maio, agosto e outubro foram programadas 470, 417 e 391 cirurgias, respectivamente, sendo suspensas $73,63,97$, apontando taxas de suspensão de $15,53,15,1$ e $24,80 \%$. A presente pesquisa

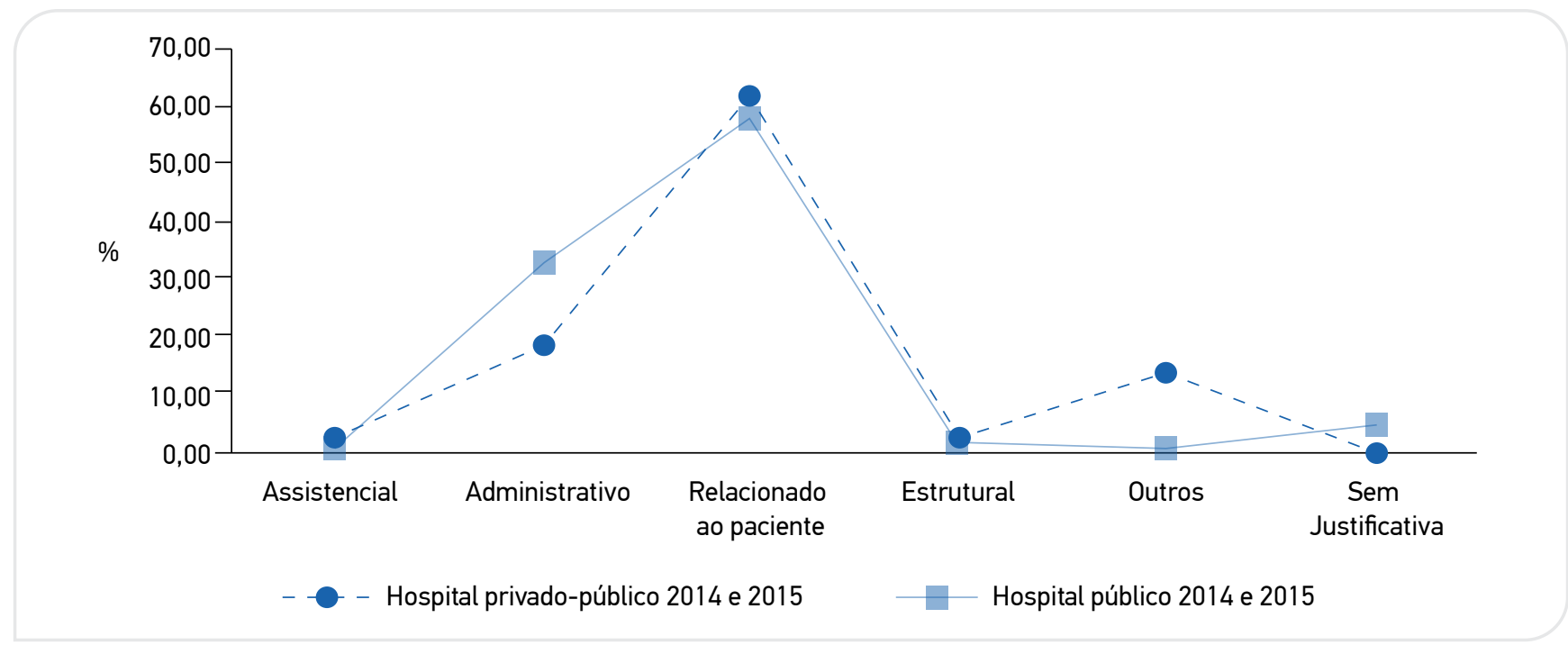

Figura 1. Comparativo dos fatores que causaram suspensão cirúrgica no hospital privado-público e no hospital público, nos anos 2014 e 2015.

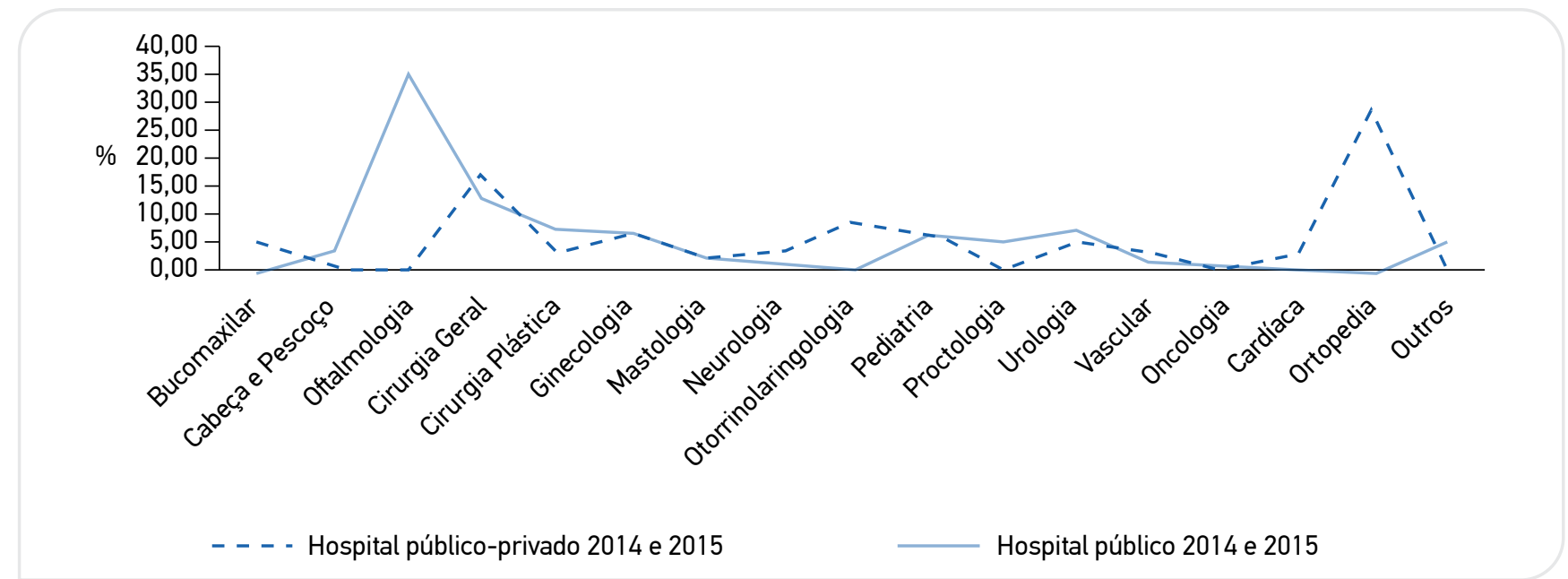

Figura 2. Comparativo das taxas de cancelamento de cirurgias no hospital privado-público e no hospital público, segundo especialidades médicas, nos anos 2014 e 2015. 
difere do estudo, pois este exibiu elevação entre o número de cancelamentos e a taxa de suspensão ${ }^{5}$.

Um estudo revela que $42,12 \%$ das suspensões foram sem justificativas e avisos não encontrados, o que demonstra falha na programação do mapa cirúrgico, já que algumas equipes cirúrgicas, para garantir a reserva de $\mathrm{SO}$ ou de equipamentos, preenchem o aviso de cirurgia com nome fictício de um paciente. Esse dado assemelha-se com o desta pesquisa, pois se trata de ações que interferem diretamente no quantitativo das cirurgias programadas, aumentam a frequência da taxa de suspensão e prejudicam a qualidade do serviço².

De acordo com a literatura, a não realização da cirurgia pode ocorrer por falha nas visitas pré-operatórias, construção do mapa cirúrgico com muita antecedência, falta de programação do cirurgião, agendamento de paciente fictício para assegurar $\mathrm{SO}$ e erro no agendamento. Todos esses motivos apresentam resolutividade e podem interferir na redução das taxas de suspensão. Diante disso, o preenchimento adequado dos motivos de substituição no mapa cirúrgico pode contribuir para a conscientização dos profissionais, por meio do conhecimento das causas que levam à suspensão?

Em equivalência com esta pesquisa, outro estudo enfatiza que a informatização do agendamento cirúrgico pode implementar a assistência no CC e colaborar para que não aconteça perda de avisos de cirurgias, otimizando as atividades $^{10}$. Nesse contexto, o CC do hospital público estudado necessita ter melhor planejamento e registro para o controle das cirurgias programadas, suspensas e realizadas.

Uma das medidas é o controle das cirurgias marcadas para o mesmo dia. Estas devem ser registradas e contabilizadas no banco de dados eletrônico da instituição como cirurgias programadas, de modo a se obter taxa de suspensão mais real. Alguns autores que defendem a informatização do agendamento cirúrgico e a necessidade de que todas as cirurgias suspensas devam ser remarcadas para o mesmo dia ou semana destacam a importância do papel do enfermeiro no planejamento e no gerenciamento da rotina do $\mathrm{CC}^{7}$.

Com base na literatura, a taxa de suspensão poderia ser reduzida com processo de intervenção. Uma medida adotada seria a implantação da sistematização da assistência de enfermagem perioperatória (SAEP), pois possibilitaria, além da avaliação pré-operatória do paciente, a comunicação intrassetorial e profissional, para garantir a continuidade do cuidado durante a internação8.

A pesquisa em evidência aponta que não houve semelhança entre o quantitativo das suspensões e o sexo biológico dos pacientes, uma vez que os hospitais apresentaram proporção inversa. Em divergência com esta pesquisa, um estudo enfatiza a diferença entre o quantitativo de cirurgias suspensas em relação ao sexo, pois foram suspensas $298(57,09 \%)$ cirurgias em pacientes do sexo masculino e $224(42,91 \%)$ do feminino ${ }^{5}$.

Com a presente pesquisa, foi possivel identificar que os principais fatores causadores das suspensões, em ambos os hospitais, foram: não comparecimento do paciente $(48,8 \%)$, falta de condições clínicas do paciente $(11,4 \%)$, falta de médicos $(6,8 \%)$ e falta de material $(6,1 \%)$. Destacam-se a falta de tempo hábil $(12,5 \%)$ no hospital privado-público e a falta de medicação $(7,64 \%)$ no hospital público.

Essa predominância do não comparecimento do paciente se dá pelo fato de o indivíduo não comparecer na instituição e pela desistência do procedimento cirúrgico. A suspensão por falta de tempo hábil, um dos fatores que apareceram no hospital privado-público, ocorre no decorrer da programação cirúrgica, quando as cirurgias prolongam o tempo de realização, ocasionando o atraso de algumas cirurgias e a suspensão de outras, por falta de tempo no dia.

Semelhantemente à pesquisa, um estudo revela ter encontrado no mapa cirúrgico todas as cirurgias suspensas com seus respectivos motivos, e verificou-se que, das 584 cirurgias suspensas classificadas como outros motivos, $100 \%$ dos casos foram ocasionados pelo avanço de horário da cirurgia anterior ${ }^{9}$.

Assim como esta pesquisa, um estudo salienta que as principais causas das suspensão estavam ligadas ao paciente $(22,7 \%)$, tais como: não comparecimento ou atraso $(22,4 \%)$ e desistência $(0,3 \%)$, sendo a terceira maior causa de cancelamento as condições clínicas do paciente $(20,8 \%)$. O segundo grupo mais frequente de suspensão cirúrgica é representado pelas condições institucionais, em razão de problemas com recursos materiais $(8,6 \%)$, erros no agendamento $(5,8 \%)$, impossibilidade de comparecimento do cirurgião (6,3\%) e alteração de data $(3,2 \%)^{11}$.

Em estudo feito em um hospital de São Paulo, por quatro anos, foram programados 22.788 procedimentos e 3.121 foram cancelados, sendo a taxa de suspensão de $13,6 \%$. Os principais fatores para a causa de suspensões foram: ausência do paciente $(33,8 \%)$, condições clínicas desfavoráveis $(20,0 \%)$, falta de tempo hábil (5,5\%), falta de vaga na UTI (4,9\%) e falta de materiais e equipamentos $(4,6 \%)^{3}$.

Já em estudo desenvolvido no período de dez meses com 6.926 cirurgias agendadas, 2.339 foram suspensas, com taxa de $33,8 \%$. Os principais motivos para suspensão foram: cancelamentos injustificados (30,1\%), representados pelas - cirurgias que apresentavam a descrição "sem motivo"; e cirurgias que foram substituídas por —; outros motivos $(25,0 \%)$, como 
avanço de horário, erro no agendamento ou desistência do paciente. Os motivos administrativos $(21,8 \%)$ foram representados pela falta de prontuários, ausência de funcionários, procedimento de urgência em outro paciente no mesmo horário, deficiência de materiais e equipamentos, cirurgias realizadas anteriormente e pacientes sem indicação cirúrgica9 .

O estudo citado anteriormente apresentou elevada taxa de suspensão $(33,8 \%)$ e diferença dos motivos das suspensões. Esse contexto pode ser reflexo da falta de planejamento das ações que envolvem todo o processo da programação cirúrgica, bem como da falta de dados para caracterizar os reais motivos da suspensão cirúrgica ou o déficit de profissionais na instituição?.

A falta do paciente merece investigação, pois não se sabe o motivo do não comparecimento. Algumas cirurgias são agendadas com bastante antecedência e não existe um serviço de busca ativa para confirmar a presença do usuário na cirurgia ${ }^{10}$. No entanto, os pacientes não comparecem à cirurgia pelo desconhecimento da data, o que revela falha na comunicação entre usuários, instituição e profissionais ${ }^{3}$.

Diante desses fatores, é necessário que as instituições adotem medidas para o controle da programação cirúrgica e desenvolvam um planejamento para melhoria da qualidade da assistência e redução das taxas de suspensão de cirurgias.

Sendo assim, estudo ressalva o papel do enfermeiro na dinâmica da rotina do CC, pois esse profissional contribui com medidas e estratégias que proporcionam assistência de enfermagem de maior qualidade e humanizada. A visita pré-operatória é uma forma de esclarecer o paciente e a sua família acerca do procedimento, desde a internação até a alta, e de passar confiança em seu cuidado ${ }^{7}$.

Em relação aos fatores administrativos, o cancelamento cirúrgico por falta de material pode evidenciar falha de gestão da instituição. No que se refere aos recursos humanos, foi visto que a ausência se deu por causa do não comparecimento do médico na instituição. Em concordância com esta pesquisa, foram identificadas $179(8,14 \%)$ suspensões por motivos administrativos, sendo a deficiência de recursos materiais $(93 / 52 \%)$ e de recursos humanos (25/14\%) os motivos mais frequentes ${ }^{8}$.

Com a avaliação, percebe-se que o não comparecimento do paciente e a falta de condições clínicas se destacam, porém os outros fatores merecem atenção, especialmente a área de ortopedia, uma vez que um percentual das suas suspensões foi em razão da falta de material essencial para o procedimento cirúrgico. Essa falta de material ocorre porque alguns materiais utilizados na cirurgia ortopédica são específicos e há falta de estoque dos fornecedores, bem como por algum motivo em nível de gestão financeira.
Diante dos resultados desta pesquisa, as especialidades de ortopedia, pediatria e urologia apresentaram redução do cancelamento no ano de 2015, enquanto para as especialidades de cirurgia geral, otorrinolaringologia e ginecologia houve aumento na taxa de suspensão. Semelhantemente a esta pesquisa, um estudo realizado no período de três meses destaca, entre as especialidades médicas com maior frequência de suspensão, a otorrinolaringologia (20,5\%), seguida da cirurgia geral $(15,7 \%)$ e da oftalmológica $(10,9 \%)$. Otorrinolaringologia e cirurgia geral mantiveram elevados índices de cancelamento durante os meses de setembro e novembro. Em contrapartida, a oftalmologia apresentou elevado número de suspensão apenas no mês de setembro ${ }^{3}$.

As especialidades que apresentaram as maiores frequências de suspensão foram: ortopedia (18,4\%), gastrocirurgia (16,1\%), oftalmologia $(10,6 \%)$ e otorrinolaringologia $(10,1 \%)^{5}$. Autores de outro estudo verificaram que a urologia e a ortopedia estão entre as especialidades com maiores taxas de suspensões ${ }^{8}$. Já em uma pesquisa feita em um hospital universitário do estado do Paraná, na qual se verificou que, das 2.828 cirurgias programadas, 522 foram suspensas, com taxa de suspensão de $18,45 \%$, a especialidade com o maior índice de cancelamento foi a cirurgia geral $(25,48 \%)^{5}$.

De acordo com os achados, a pesquisa apresenta semelhança com os estudos citados, pois no hospital privado-público a ortopedia foi a especialidade com maior taxa de suspensão, seguida da gastrocirurgia ${ }^{4}$. No entanto, é necessário levar em consideração que, no hospital privado-público, a cirurgia geral inclui a gastrocirurgia. Em outros estudos, a ortopedia está entre as especialidades de maior incidência de suspensão ${ }^{8}$ e a cirurgia geral evidencia o maior índice de suspensões ${ }^{5}$.

Já em relação à oftalmologia, a especialidade desponta com os maiores índices de suspensão ${ }^{10}$. No hospital público é a especialidade de maior suspensão cirúrgica, seguida da cirurgia geral. Desse modo, destaca-se similaridade nas especialidades mais incidentes e diferença na proporção em que aparecem. As outras especialidades também são destaques encontrados por outros autores, sugerindo semelhança entre os estudos, com ênfase para urologia ${ }^{8}$ e otorrinolaringologia ${ }^{10}$.

Estudo realizado em instituição de saúde em São Paulo evidencia que o cancelamento de cirurgias, em algumas situações, decorre da falta de planejamento. As ações desenvolvidas no centro de material e esterilização (CME), por ser o setor responsável pelo fornecimento de materiais para atender aos diversos procedimentos anestésico-cirúrgicos, repercutem diretamente na dinâmica do CC. Assim, a falha em algum dos processos pode acarretar atraso de cirurgias e até mesmo o seu cancelamento. Dessa forma, o evento merece atenção 
da equipe envolvida na realização do procedimento, principalmente quando os motivos são potencialmente evitáveis ${ }^{12}$.

É importante destacar, diante do contexto, que a enfermagem apresenta papel fundamental no planejamento e no controle dos procedimentos cirúrgicos e pode contribuir com estratégias para a redução das suspensões, além de fornecer ao paciente e à sua família assistência de qualidade. Além disso, pode buscar medidas de organização dos serviços das instituições de saúde, como controle da programação cirúrgica e confirmação da cirurgia, visando à melhor qualidade do serviço da instituição.

No processo da pesquisa, houve algumas limitações, como a dificuldade de acesso ao arquivo dos hospitais e a falta do preenchimento completo do mapa cirúrgico. A pesquisa evidencia que todo o processo necessita de gerenciamento e planejamento das ações para obter redução da suspensão cirúrgica. Sendo assim, será relevante uma investigação com foco na análise das medidas gerenciais, administrativas e assistenciais envolvidas no processo de qualidade da assistência e na redução dos indicadores e da taxa de suspensão de procedimentos anestésico-cirúrgicos.

\section{CONCLUSÃO}

Com esta pesquisa, constataram-se variações no quantitativo das cirurgias programadas e canceladas, revelando desigualdade nas taxas de suspensão cirúrgica entre os hospitais estudados, sendo a taxa do hospital público $(42,47 \%)$ duas vezes maior que a do hospital privado-público $(23,13 \%)$.

Os principais fatores que causaram suspensão nos hospitais pesquisados foram: falta do paciente e falta de condições clínicas, quando relacionados ao paciente; e falta de material e de médicos, no tocante aos administrativos. Já as principais especialidades cirúrgicas com maiores taxas de suspensão no hospital privado-público foram a ortopedia e a cirurgia geral; e no hospital público, a oftalmologia e a cirurgia geral. Nesse contexto, os hospitais apresentam diferenças entre as principais especialidades de suspensão e semelhança quando se trata da cirurgia geral.

De acordo com os resultados, a incidência de cancelamento de cirurgias nas instituições apresenta grande importância assistencial e administrativa, como também na área cientifica, por favorecer identificação dos indicadores de qualidade, redução da taxa de suspensão e busca da melhoria da qualidade da assistência em saúde.

Para isso, a enfermagem precisa exercer papel essencial na construção de medidas que promovam a melhoria do controle do quadro cirúrgico, como manter a organização do setor com recursos materiais e humanos e monitorar a equipe e a programação cirúrgica, evitando as suspensões. Dessa forma, utilizam-se ferramentas de gestão de qualidade, com a finalidade de promover aumento da qualidade gerencial, assistencial e humanização das relações com os pacientes. As ações de enfermagem podem melhorar a assistência prestada ao paciente, estabelecendo planejamento individualizado e acompanhamento desde a internação até a alta hospitalar.

Assim, as instituições necessitam lançar estratégias para o controle da programação cirúrgica. Um método é melhorar a comunicação da instituição com o usuário, com planejamento e acompanhamento semanal da programação cirúrgica, para que possa, com antecedência, entrar em contato com o paciente para confirmação cirúrgica, bem como realizar a consulta pré-anestésica.

\section{REFERÊNCIAS}

1. Ávila MAG, Bocchi SCM. Confirmação de presença de usuário à cirurgia eletiva por telefone como estratégia para reduzir absenteísmo. Rev Esc Enferm USP. 2013;47(1):193-7. http://dx.doi.org/10.1590/ S0080-62342013000100024

2. Morgan W, Bernardino E, Wolff LDG. Implicações do cancelamento de cirurgias em centro cirúrgico: estudo descritivo-exploratório. Online Braz J Nurs. 2010 [acessado em 10 jan. 2018];9(1):1-14. Disponível em: http://www.objnursing.uff.br/index.php/nursing/ article/view/j.1676-4285.2010.2591/585

3. Sodré RL, El Fahl MAF. Cancelamento de cirurgias em um hospital público na cidade de São Paulo. RAS. 2014;16(63):67-70. http://dx.doi. org/10.5327/Z1519-1672201400630005
4. Ávila MAG, Gonçalves IR, Martins I, Moyses AM. Cancelamento de cirurgias: uma revisão integrativa da literatura. Rev SOBECC. 2012;17(2):39-47.

5. Pinheiro SL, Vasconcelos RO, Oliveira JLC, Matos FGOA, Tonini NS, Alves $\mathrm{DCl}$. Taxa de cancelamento cirúrgico: Indicador de qualidade em hospital universitário público. Rev Min Enferm. 2017;21:e1014. http://www.dx.doi.org/10.5935/1415-2762.20170024

6. Botazini NO, Carvalho R. Cancelamento de Cirurgias: uma revisão integrativa da literatura. Rev SOBECC. 2017 [acessado em 5 de nov. 2019];22(4):230-44. Disponível em: http://docs.bvsalud. org/biblioref/2017/12/876633/sobecc-v22n4_pt_230-244.pdf. http://www.dx.doi.org/10.5327/Z1414-4425201700040008 
7. Botazini NO, Toledo LD, Souza DMST. Cirurgias eletivas: cancelamentos e causas. Rev SOBECC. 2015;20(4):210-9. http://www.dx.doi. org/10.5327/Z1414-4425201500040005

8. Barbosa MH, Miranda DMG, Vieira de Andrade E, De Mattia AL. Análise da suspensão de cirurgias em um hospital de ensino. Rev Elet Trim Enferm. 2012 [acessado em 24 jan. 2018];(26)174-83. Disponível em: http://scielo.isciii.es/pdf/eg/v11n26/pt_administracion2.pdf

9. Gomes JRAA, Franco RVB, Morais DSVD, Barbosa BC. Fatores determinantes para suspensões de cirurgias eletivas em um hospital do distrito federal. Rev SOBECC. 2018;23(4): 184-8. http://www.dx.doi. org/10.5327/Z1414-4425201800040003
10. Macedo JM, Kano JA, Braga EM, Garcia MA, Caldeira SM. Cancelamento de cirurgias em um hospital universitário: causas e tempos de espera para novo procedimento. Rev SOBECC. 2013;18(1):26-34.

11. Carvalho TA, Sobral CB, Marinho PML, Llapa-Rodriguez EOO, Campos MPA. Suspensão de cirurgias em um hospital universitário. Rev SOBECC. 2016;21(4):186-91. http://www.dx.doi.org/10.5327/ Z1414-4425201600040002

12. Tamiasso RSS, Santos DC, Fernandes VDO, loshida CAF, Turrini RNT. Ferramentas de gestão de qualidade como estratégias para redução do cancelamento e atrasos de cirurgias. Rev SOBECC. 2018;23(2):96-102. http://www.dx.doi.org/10.5327/Z1414-4425201800020007 